\title{
The Research of Cylindrical Billets Induction Heating Based on Finite Difference Method
}

\author{
Zhang Qingxin, Zhu Cuiyu, Tao Yong, Cui Zhanbo \\ Automation Department, Shenyang Aerospace University, Shenyang, 110136, China \\ zhy9712@163.com
}

Key words: Induction Heating; Finite Difference; Temperature Field; Electromagnetic field

\begin{abstract}
The induction heating temperature and electromagnetic field coupling model has been established based on the principle of induction heating and finite difference method. The suitable parameters are set up according to actual size of billet. Numerical simulations are performed according to the mathematics model above with Visual C++ programs.
\end{abstract}

\section{The Background of Induction Heating Technique}

Induction heating gets really application as a new technology in recently 30 years. It is widely used for the shortage of resources, which promote the development of induction heating. It has the following features: [1] it has accurate heating depth and areas, and it's easy to be controlled. [2] It's convenient to realize high-power heating, and it has fast heating speed, high efficiency and low energy. [3] Induction heating takes the noncontact heating method that couldn't easily mix impurities in billet. [4] There's little burning loss or oxide skin on billet. [5] Induction heating operation environment fits the environmental standard.

\section{The Principle of Induction Heating}

The principle of the metal billet induction heating working is that the heated metal billet is around on a group of induction coil outside. When a certain vibration frequency ac-current goes through the coil, the same frequency alternate magnetic flux was generated. The equipment of induction heating is showed in figure 1.

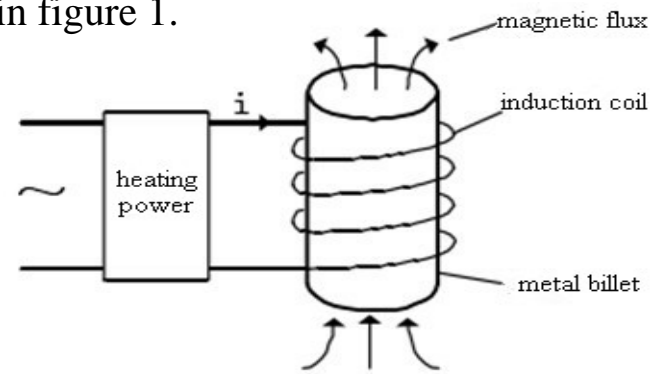

Figure 1. The equipment of induction heating

Induction heating principle can be described with electromagnetic induction theorem and Joule-Lenz's law. The electromagnetic induction theorem is: when magnetic flux $\phi$ passes through the plane that is limited by each closed loop changes as time, the closed loop can produce electromotive force $\mathrm{E}$ :

$$
E=-\frac{d \phi}{d t} \text {. }
$$

Electromotive force makes the billet produce eddy current, moreover Joule heat is produced. The following is the expression of Joule-Lenz's law:

$$
q=i^{2} R t \text {. }
$$

$\varphi 1600 \times 2000 \mathrm{~mm}$ cylindrical billet is studied in this article and the billet material uses the No.45 steel. To make the billet remain effectively hardness and hardening depth, the work surface should have a certain depth austenitizing and cores remain in the phase transition temperature below point. 
Research shows that the billet has phase transition point in induction heating, the phase change point temperature of No.45 steel is $750^{\circ} \mathrm{C}$. The eddy current concentrates on the surface when the billet temperature is below the phase transition point temperature. Eddy current distribution changed when the billet temperature is higher than the phase transition point's. The following expression shows the relationship between heating frequency and "pervious bed depth"

$$
\delta=\sqrt{\frac{2 \rho}{\mu_{r} \mu_{0} f}} .
$$

Where $\delta$ is the depth of pervious bed, $\rho$ is resistivity $(\Omega m), \mu_{r}$ is relative magnetic permeability, $\mu_{0}=4 \pi \times 10^{-7} \mathrm{~T} / \mathrm{mA}$ is space permeability, $f=50 \mathrm{~Hz}$ is alternating current.

The current density distribution is not uniform, supposing $I_{0}$ is surface current, the current density from surface to inner is:

$$
I_{x}=I_{0} e^{-x / \delta} \text {. }
$$

Where $x$ is the distance from Lateral surface of billet, $x=R-r . I_{x}$ is eddy density of the point at the distance from axis $r$.

\section{The Analysis of Induction Heating Temperature Field}

The eddy current is taken as internal heat source heating the billet. Firstly according to the joule's law the value can be solved. Differential equation of heat conduction under column coordinates is:

$$
\frac{1}{r} \frac{\partial}{\partial r}\left(r k \frac{\partial T}{\partial r}\right)+\frac{1}{r^{2}} \frac{\partial}{\partial \theta}\left(k \frac{\partial T}{\partial \theta}\right)+\frac{\partial}{\partial z}\left(k \frac{\partial T}{\partial z}\right)+q=\rho c \frac{\partial T}{\partial t} .
$$

Where $q$ is internal intensity $\left(\mathrm{W} / \mathrm{m}^{3}\right), \rho$ is density of the billet, $c$ is specific heat $\left(\mathrm{J} / \mathrm{kg}^{\circ} \mathrm{C}\right)$. In this article, cylindrical billet is studied; axis-symmetric load is applied, so the billet can be similar to two-dimensional. Then there is the following expression:

$$
\frac{\partial T}{\partial \theta}=0
$$

Substituting the expression into(6),

$$
\frac{1}{r} \frac{\partial}{\partial r}\left(r k \frac{\partial T}{\partial r}\right)+\frac{\partial}{\partial z}\left(k \frac{\partial T}{\partial z}\right)+q=\rho c \frac{\partial T}{\partial t} \text {. }
$$

For transient heat conduction problem the basic means of numerical analysis is the finite difference equation instead of differential equation. In view of the partial derivative of $T(\xi, \tau)$ at the point $(\xi, \tau)$, where $\xi$ and $\tau$ are mutual independent variables, and taking the method of backward finite difference:

$$
\begin{aligned}
& \left(\frac{\partial T}{\partial \xi}\right)_{\xi, \tau} \approx \frac{1}{\delta \xi}[T(\xi+\delta \xi, \tau)-T(\xi, \tau)] . \\
& \left(\frac{\partial T}{\partial \tau}\right)_{\xi, \tau} \approx \frac{1}{\delta \tau}[T(\xi, \tau+\delta \tau)-T(\xi, \tau)] .
\end{aligned}
$$

According to definition of derivative, when $\delta \xi$ and $\delta \tau$ tending to 0 , the expressions above can change to strict equality.

Applying the expression above into cylinder conduction model the passive unsteady two-dimensional difference equation can be got in cylindrical coordinate system:

$$
\begin{aligned}
T_{(i, j)}^{n+1}= & \frac{\lambda}{\rho c} \cdot \frac{\Delta t}{\Delta z^{2}} \cdot T_{(i+1, j)}^{n}+\frac{\lambda}{\rho c} \cdot \frac{\Delta t}{\Delta z^{2}} \cdot T_{(i-1, j)}^{n}+\frac{\lambda}{\rho c}\left(\frac{\Delta t}{\Delta r^{2}}+\frac{\Delta t}{r \Delta r}\right) T_{(i, j+1)}^{n}+\frac{\lambda}{\rho c} \cdot \frac{\Delta t}{\Delta r^{2}} \cdot T_{(i, j-1)}^{n} . \\
& +\left(1-\frac{\lambda}{\rho c} \cdot \frac{2 \Delta t}{\Delta r^{2}}-\frac{\lambda}{\rho c} \cdot \frac{\Delta t}{r \Delta r}-\frac{\lambda}{\rho c} \cdot \frac{2 \Delta t}{\Delta z^{2}}\right) \cdot T_{(i, j)}^{n}
\end{aligned}
$$

Where $i$ is the number of the layers from inner to surface. $j$ is the number of layers from underside to top surface. 
According to the stability theory of explicit difference equation, the stable expression need satisfy condition that the coefficient of $T_{(i, j)}^{n}$ is positive number, and the time interval need satisfy the following expression:

$$
\Delta t \leq{\frac{\Delta r^{2} \Delta z^{2}}{\frac{\lambda}{\rho c}\left(2 \Delta z^{2}+\frac{\Delta r \Delta z^{2}}{r}+2 \Delta r^{2}\right)}}^{\circ}
$$

For simple calculation, when $r=\Delta r$, that are nearest nodes from axis, the right-hand of the expression above can get minimum:

$$
\Delta t \leq \frac{\Delta r^{2} \Delta z^{2}}{\frac{\lambda}{\rho c}\left(3 \Delta z^{2}+2 \Delta r^{2}\right)} .
$$

From the expression (12), it can get the calculating maximum time interval of explicit difference equation.

Upper and lower surface of the billet have radiation and convection. Due to the surface temperature of billet is very high, the heat air exchange in the surface can be ignored. Radiation heat transfer of Stepan- Boltzmann's expression is:

$$
q=\varepsilon \sigma A\left(T_{E}^{4}-T^{4}\right) \text {. }
$$

Where $\varepsilon$ is radiation coefficient, $\varepsilon=0.7, \sigma=5.6697 \times 10^{-8} \mathrm{~W} / \mathrm{m}^{2} \cdot K^{4}$ is constant, $A$ is the contact area between billet and air.

The physical parameters of the billet change with increasing temperature in the process of induction heating. Table 1 . lists the heat capacity, density, relative permeability, thermal conductivity.

Table 1. The parameter of No. 45 steel

\begin{tabular}{llllll}
\hline $\begin{array}{l}\text { Temperature } \\
\left({ }^{\circ} \mathrm{C}\right)\end{array}$ & $\begin{array}{l}\text { Density } \\
\left(\mathrm{kg} / \mathrm{m}^{3}\right)\end{array}$ & $\begin{array}{l}\text { Capacity } \\
(\mathrm{J} / \mathrm{kgK})\end{array}$ & $\begin{array}{l}\text { Resistivity } \\
\left(\times 10^{-6} \Omega \mathrm{m}\right)\end{array}$ & $\begin{array}{l}\text { Conductivity } \\
(\mathrm{W} / \mathrm{mK})\end{array}$ & $\begin{array}{l}\text { Relative } \\
\text { permeability }\end{array}$ \\
\hline 100 & 7773.4 & 480 & 0.254 & 43.53 & 195 \\
200 & 7740.0 & 498 & 0.339 & 40.44 & 186.6 \\
500 & 7640.0 & 615 & 0.656 & 34.16 & 154.9 \\
750 & 7600.0 & 986 & 1.019 & 26.20 & 11 \\
800 & 7600.0 & 806 & 1.080 & 26.49 & 1 \\
100 & 7600.0 & 602 & 1.200 & 24.02 & 1 \\
\hline
\end{tabular}

Radial grids of the billet are divided according to electromagnetic theory of induction heating, there are 2 to 3 grids are divided in eddy current layer. 40 layers are divided evenly on billet. This paper studies the heating depth of radial mainly, and then 20 layers are divided evenly on axial.

\section{The Numerical Simulation of Induction Heating}

Firstly the billet's surface is heated to $1050^{\circ} \mathrm{C}$ at the full power. Then the surface temperature is controlled at $1050 \pm 30^{\circ} \mathrm{C}$ by adjusting the power rate. When the surface temperature exceeds the upper limit, the power dropped to $80 \%$; it is below the lower limit, the power is adjusted to $120 \%$. The target point temperature was heated to $850{ }^{\circ} \mathrm{C}$. The calculation uses Visual C++ programming for numerical simulation, and save the results in .csv file. Then Matlab read the file and plot temperatures change contracted figures.

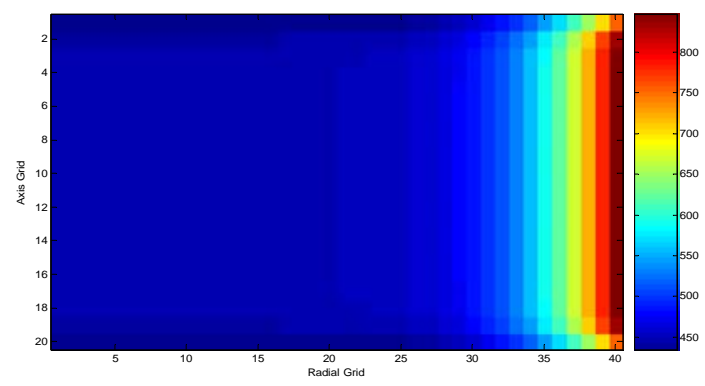

Figure 2. The temperature distribution after full power heating

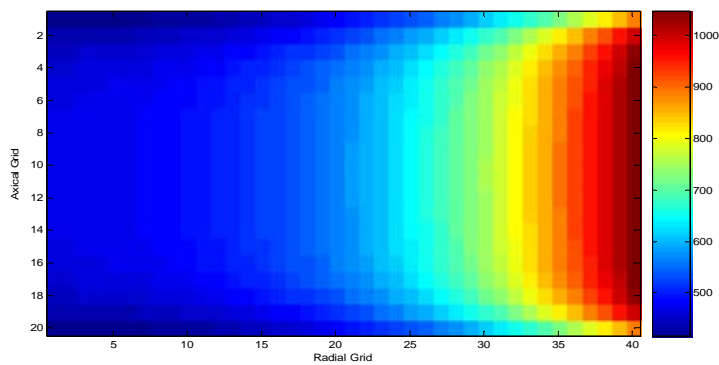

Figure 3. The temperature distribution after adjusting power 
The figure2 is the temperature distribution after full power of $3500 \mathrm{KW}$ heating, which can make the side surface temperature reach to $1050^{\circ} \mathrm{C}$. The radiation distributes on the upper, lower and side surface, and the temperature of axis tends to decrease from the center of symmetry to the upper and lower surface.

In order to control surface temperature not to be too high, the power needs to be adjusted to limit the maximum temperature of the induction heating. By adjusting the proportion of power from top to bottom the target point can reach to $850^{\circ} \mathrm{C}$. The temperature distribution after adjusting power is showed in figure 3.

The point of side surface center and target point's heating temperature change from beginning to the end in Figure4.

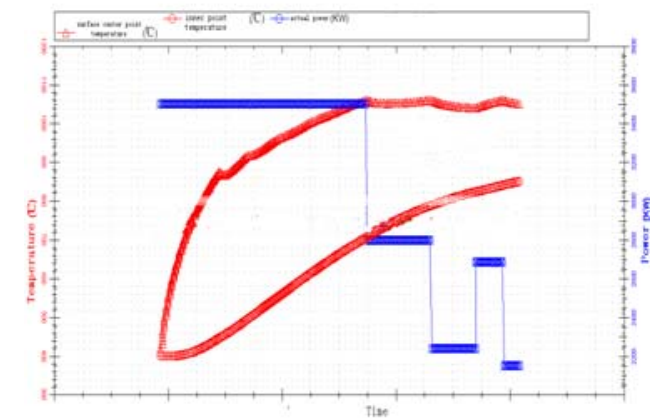

Figure 4.Power and target point, side surface temperature

\section{Experimental Results and Analysis}

By numerical simulation, it can be seen that surface temperature rise rapidly at the beginning of the heating, but the target point's temperature rises a little slowly. After the billet's temperature reaching to the phase transition point, the surface temperature and the target point's both rise slowly down.

The billet is heated at full power. It needs 2790 s when its surface temperature rises from $450^{\circ} \mathrm{C}$ to $1050{ }^{\circ} \mathrm{C}$. The time is $1720 \mathrm{~s}$ when adjusting power control the side surface temperature at $1050 \pm 30^{\circ} \mathrm{C}$ and the target temperature is $850^{\circ} \mathrm{C}$.

In the analysis of the principle of induction heating process, ignoring the impact of secondary factors of air convection on the temperature of the billet surface temperature will cause a certain deviation.

\section{References}

[1] Donald Pirts, Leighton Sissom. Schaum's Outline of Theory and Problems of Heat Transfer [J]. NewYork: McGraw-Hill Companies, 1998. 1-4, 78-82.

[2] Yuehong Zhang. Experimental and Simulation Studies of The Temperature Field of Induction Heating [J]. Technology and Development Enterprise, 2010, (4):44-46.

[3] Jiquan Liu. Induction Heating of the Thermal Calculation Model [J]. Heavy Castings and Forging, 2003, (3):16-21.

[4] Huaiyu Sun, Zhumin Wang. Two-dimensional Heat Transfer Investigation in Algorithm Analysis and General Calculation procedure. Chemical Engineer, 2006(2):17-18.

[5] Xian Ze, Yajing Xiao, Qianfeng Shi. The Development of Induction Heating Technology, Technology and Equipment, 2010(3):62-63. 